\title{
A retrospective study of trifluridine/tipiracil in pretreated metastatic colorectal cancer patients in clinical practice
}

\author{
Beatrice Borelli ${ }^{1}$, Gemma Zucchelli ${ }^{1}$, Daniele Rossini ${ }^{1}$, Chiara Cremolini ${ }^{1}$, Carlotta \\ Antoniotti ${ }^{1}$, Roberto Moretto ${ }^{1}$, Federica Marmorino ${ }^{1}$, Camilla Colombo ${ }^{1}$, Francesca \\ Vannini ${ }^{1}$, Laura Delliponti ${ }^{1}$, Mario Spione ${ }^{2}$, Elena Ongaro ${ }^{3}$, Gianluca Masi ${ }^{1}$, Isa Maura \\ Brunetti ${ }^{1}$, Elisabetta Pfanner ${ }^{1}$, Lisa Salvatore ${ }^{1}$ \& Alfredo Falcone*,1 \\ ${ }^{1}$ Unit of Medical Oncology, Azienda Ospedaliero-Universitaria Pisana, Department of Translational Research \& New Technologies in \\ Medicine, University of Pisa, Pisa, Italy \\ ${ }^{2}$ Medical Affairs, Servier Italia SpA, Roma, Italy \\ ${ }^{3}$ Department of Oncology, Azienda Sanitaria Universitaria Integrata S. Maria della Misericordia, Udine, Italy \\ *Author for correspondence: alfredo.falcone@med.unipi.it
}

\begin{abstract}
Aim: RECOURSE trial has shown a benefit for trifluridine/tipiracil (also known as TAS-102) over placebo in chemorefractory metastatic colorectal cancer (mCRC) patients. We evaluated efficacy and safety of patients treated with trifluridine/tipiracil in compassionate use. Patients \& methods: RETROTAS is a retrospective study designed to evaluate efficacy and safety of trifluridine/tipiracil in $\mathrm{mCRC}$ patients previously exposed or not a candidate to all available drugs. Results: A total of $85 \mathrm{mCRC}$ patients were included. Our data showed that $37.6 \%$ of patients achieved disease control. Median progression-free survival was 2.3 months; at 16 months follow-up, median overall survival was 9.2 months. Conclusion: This real-life experience confirms the safety and efficacy of trifluridine/tipiracil, resulting as a valid option in chemorefractory $\mathrm{mCRC}$ treatment scenario.
\end{abstract}

First draft submitted: 24 December 2017; Accepted for publication: 16 February 2018; Published online: 9 March 2018

Keywords: chemorefractory $\bullet$ chemotherapy $\bullet$ fluoropyrimidine $\bullet \mathrm{mCRC} \bullet$ real-life $\bullet$ refractory colorectal cancer $\bullet$ TAS-102 • trifluridine/tipiracil

Heavily pretreated metastatic colorectal cancer (mCRC) patients deemed refractory to all active drugs (fluoropyrimidines, irinotecan, oxaliplatin, anti-VEGF and anti-EGFR agents, in $R A S$ wild-type patients), present a very poor prognosis and have limited chances of treatment $[1,2]$. For $\mathrm{mCRC}$ patients who have progressed on these therapies, currently, we have three major options above participation in clinical trials: regorafenib, trifluridine/tipiracil (TAS102) or best supportive care [3]. Regorafenib, an oral multitarget tyrosine kinase inhibitor with anti-angiogenic activity, was until few years ago the only drug to demonstrate superiority in terms of progression-free survival (PFS) and overall survival (OS) against placebo in a Phase III trial in later lines [4].

Trifluridine/tipiracil was approved by the Japanese Ministry of Health in March 2014, US FDA in September 2015 and subsequently by the EMA. Trifluridine/tipiracil is a novel oral nucleoside antitumor agent consisting of trifluridine (FTD), a fluorinated thymidine analog, and tipiracil hydrochloride, a thymidine phosphorylase inhibitor. Thymidine phosphorylase inhibitor is essential to prevent FTD rapid inactivation, improving plasma levels and the related antitumor activity of FTD; moreover it seems also to have a direct antiangiogenic role [57]. FTD can be misincorporated into the double helix of DNA after its phosphorylation by thymidine kinase causing DNA strand breaks and cell apoptosis in a direct and irreversible way; this mechanism of action is very different from 5-FU and capecitabine, which reversibly inhibit the thymidylate synthase blocking the production of deoxythymidine monophosphate [8-10].

On the basis of preclinical data [11-13] and Phase I/II trials [14-17], two randomized, double-blind, placebocontrolled Phase III trials investigated the potential role of trifluridine/tipiracil in refractory mCRC patients. In the international RECOURSE trial, median OS (mOS) was 7.1 months in trifluridine group and 5.3 months in the 
placebo group (hazard ratio [HR]: 0.48; 95\% CI: $0.41-0.57$; $<<0.001$ ), with $44.0 \%$ for trifluridine/tipiracil versus $16.3 \%$ for the placebo $(\mathrm{p}<0.0001)$ as disease control rate [18]. The study also reported a statistical improvement in PFS from 1.7 to 2.0 months in the trifluridine/tipiracil arm (HR: 0.48; $\mathrm{p}<0.001$ ).

In addition, the efficacy of trifluridine/tipiracil was confirmed in an mCRC Asian population by TERRA study which showed an improvement both in OS and PFS versus placebo in the primary analysis (mOS: 7.8 vs 7.1 months; HR: 0.79; 95\% CI: 0.62-0.99; $\mathrm{p}=0.035$ and median PFS 2.0 vs 1.8 months; HR: 0.43; 95\% CI: 0.34-0.53; $\mathrm{p}<0.001)$ [19]. The safety profile was similar in both studies with a good overall tolerance. The neutropenia was the most frequent grade 3/4 treatment-emergent adverse event (AE; 38\% in RECOURSE and 20.3\% in TERRA, respectively) $[18,19]$.

Considering that in Phase III randomized-controlled trials eligibility criteria are restrictive and there are often no appropriate ongoing clinical trials available, most of the chemorefractory patients have no other therapeutic options [20]; despite that, they could be able to receive new unauthorized drugs only through compassionate use [21,22].

Compassionate use is important for patients, to give them another chance; it is also important for physicians in order to obtain useful and fit data for clinical practice beyond obvious deviations from the ideal selected populations included in randomized-controlled trials [23]. Based on our single-center experience, we reported the efficacy and the safety results for a consecutive cohort of 85 chemorefractory, heavily pretreated mCRC patients treated with trifluridine/tipiracil in compassionate use in our institution.

\section{Patients \& methods}

The study population consisted of 85 patients with a histologically confirmed diagnosis of colorectal adenocarcinoma previously exposed or considered not a candidate to all available drugs and progressed during or within 3 months after the last administration of standard therapy were eligible for trifluridine/tipiracil (also known as TAS-102).

Eligibility for the study also required a written informed consensus, age $\geq 18$ years, Eastern Cooperative Oncology Group Performance Status (ECOG PS) 0-2, and adequate liver, renal and bone marrow function.

Trifluridine/tipiracil was administered with 28-day cycles, each made of 5 days of treatment followed by a 2-day rest period every week for 2 weeks, and then a 14-day rest period. A dose of $35 \mathrm{mg}$ per square meter of body surface area twice-daily was used on the basis of Phase I clinical trials. In case of toxicities, dose reduction(s) could be performed in $5 \mathrm{mg}$ per square meter steps; only three dose reductions of the medication are recommended. AEs occurring during the treatment were registered and graded according to the Common Terminology Criteria for Adverse Events v4.0 [24].

Every patient can be withdrawn from treatment in case of Response Evaluation Criteria in Solid Tumors (RECIST 1.1) [25] defined or clinical disease progression, unacceptable AEs or irreversible treatment-related grade 4 toxicity. Disease assessments were performed with contrast-enhanced CT scan every 8 weeks ( 2 cycles) of treatment.

\section{Statistical analysis}

Descriptive statistics were used to summarize data. We evaluated efficacy and activity for patients treated with trifluridine/tipiracil in terms of PFS, defined as the time to the first progression according to RECIST 1.1 criteria [25] or death, whichever came first; OS, as the time to the date of death due to any cause or, if nonprogressing patients alive at the time of analysis, of the last follow-up; objective response rate, as the proportion of patients that achieved a confirmed complete response (CR) or a partial response (PR) as best response; safety profile. All time-to-event variables were calculated from the date of first trifluridine/tipiracil administration. The Kaplan-Meier method was used to estimate PFS and OS durations with 95\% CI.

\section{Results}

\section{Study population}

A consecutive cohort of $85 \mathrm{mCRC}$ patients received trifluridine/tipiracil at U.O. Oncologia Medica 2 Universitaria - Azienda Ospedaliero - Universitaria Pisana, Santa Chiara Hospital, Pisa, Italy. This population represents a setting of chemorefractory, mainly heavily pretreated patients, with widespread metastatic disease.

Patients' baseline characteristics are summarized in Table 1. The median age of the population is 67 years, with more of 50\% (52) patients with a good clinical status (ECOG PS 0) and only 2.3\% (2) with ECOG PS 2. A total of $82 \%$ of the patients had more than one site of metastasis: liver and lung are the most represented (78.8 and $65.9 \%)$. 
Table 1. Patients' clinical and molecular characteristics.

\begin{tabular}{|c|c|c|c|}
\hline \multicolumn{2}{|l|}{ Characteristics } & \multirow{2}{*}{$\begin{array}{l}n=85 \\
47 / 38\end{array}$} & \multirow{2}{*}{$\begin{array}{l}\text { Percentage } \\
55.3 / 44.7\end{array}$} \\
\hline Gender & $\mathrm{M} / \mathrm{F}$ & & \\
\hline Age & Median (range) & $67(45-83)$ & - \\
\hline \multirow{2}{*}{ ECOG PS } & 1 & 31 & 36.5 \\
\hline & 2 & 2 & 2.3 \\
\hline \multirow[t]{6}{*}{ Sites of metastases } & Liver & 67 & 78.8 \\
\hline & Nodes & 45 & 52.9 \\
\hline & Peritoneum & 24 & 28.2 \\
\hline & Bone & 9 & 10.6 \\
\hline & Brain & 2 & 2.3 \\
\hline & Liver only & 6 & 7.0 \\
\hline Number of metastatic sites & 4 & 11 & 12.9 \\
\hline \multirow[t]{2}{*}{ Time from start of first-line treatment } & $<18$ months & 28 & 32.9 \\
\hline & $\geq 18$ months & 57 & 67.1 \\
\hline \multirow[t]{6}{*}{ Number of previous lines of treatment } & 1 & 8 & 9.4 \\
\hline & 2 & 27 & 31.8 \\
\hline & 3 & 26 & 30.6 \\
\hline & 4 & 10 & 11.8 \\
\hline & 5 & 11 & 12.9 \\
\hline & 6 & 3 & 3.5 \\
\hline $\begin{array}{l}\text { Prior systemic anticancer agents for } \\
\text { metastatic disease }\end{array}$ & Fluoropyrimidines & 84 & 98.8 \\
\hline \multirow[t]{3}{*}{ RAS status } & Wild-type & 30 & 35.3 \\
\hline & Mutated & 48 & 56.5 \\
\hline & Not available & 7 & 8.2 \\
\hline \multirow[t]{3}{*}{$B R A F$ status } & Wild-type & 71 & 83.5 \\
\hline & Mutated & 6 & 7.1 \\
\hline & Not available & 8 & 9.4 \\
\hline
\end{tabular}

The numbers of previous lines of treatment are 2, 3 and a higher number, in the 31.8, 30.6 and $28.2 \%$ of the population. A large percentage of patients had disease that had been refractory to fluoropyrimidines $(98.8 \%)$, irinotecan (95.3\%), bevacizumab (88.2\%), oxaliplatin (81.2\%), EGFR inhibitors (42.3\%) and regorafenib (24.7\%).

Treatment exposure, management \& safety profile

The number of cycles of trifluridine/tipiracil received by each patient range from 1 to 13 (Table 2). Interestingly, 22 patients $(25.9 \%)$ received at least six cycles of therapy. In respect of tolerability, 37 patients $(43.5 \%)$ had a delay in beginning their next cycle of therapy due to the occurrence of AEs. A total of 32 patients (37.6\%) required dose reduction due to AEs, with 17 patients (20.0\%) having a single dose reduction, 11 (12.9\%) having two 


\section{Table 2. Treatment exposure and management.}

\begin{tabular}{|c|c|c|c|}
\hline & & $n=85$ & Percentage \\
\hline \multirow[t]{10}{*}{ Number of cycles of treatment received } & 1 & 4 & 4.7 \\
\hline & 2 & 39 & 45.9 \\
\hline & 3 & 12 & 14.1 \\
\hline & 4 & 3 & 3.5 \\
\hline & 5 & 5 & 5.9 \\
\hline & 6 & 11 & 12.9 \\
\hline & 7 & 6 & 7.1 \\
\hline & 8 & 3 & 3.5 \\
\hline & 9 & 1 & 1.2 \\
\hline & 13 & 1 & 1.2 \\
\hline Number of cycles of treatment received & Median (range) & $2(1-13)$ & - \\
\hline Patients with cycles delayed due to AEs & Yes & 37 & 43.5 \\
\hline Patients with dose reduction due to AEs & Yes & 32 & 37.6 \\
\hline
\end{tabular}

\section{Table 3. Safety profile.}

\begin{tabular}{|c|c|c|}
\hline Grade $3 / 4$ adverse event & $n=85$ & Percentage \\
\hline Leucopenia & 13 & 15.3 \\
\hline Neutropenia & 49 & 57.6 \\
\hline Anemia & 7 & 8.2 \\
\hline Thrombocytopenia & 1 & 1.2 \\
\hline Nausea & 1 & 1.2 \\
\hline Fatigue & 3 & 3.5 \\
\hline Diarrhea & 1 & 1.2 \\
\hline Overall toxicity & 56 & 65.9 \\
\hline
\end{tabular}

reductions and five (5.9\%) having three dose reductions. Only one patient definitely discontinued the treatment due to toxicity.

Overall, 56 (65.9\%) of patients experienced at least one grade 3/4 AE during the treatment and no toxicrelated deaths were reported (Table 3). The most frequent grade 3/4 AEs were hematologic toxicities: neutropenia occurred in 49 out of 85 patients (57.6\%), anemia (8.2\%) and thrombocytopenia (1.2\%) arose less frequently. Nonhematologic grade $3 / 4$ AEs such as fatigue (3.5\%), nausea (1.2\%) and diarrhea (1.2\%) were less frequent.

\section{Activity \& efficacy}

Our data showed that 32 patients (37.6\%) achieved a disease control, while disease progression was the best response in 51 cases (60.0\%). No PR or CR was reported (objective response rate $0 \%$ ). Two patients (2.3\%) did not undergo disease reassessment: one patient died after the first cycle and one patient was early lost to follow-up. The median PFS was 2.3 months (Figure 1).

A total of 31 patients $(36.5 \%)$ did not achieve progression at 4 months from the beginning of treatment, interestingly $18(21.2 \%)$ patients were still on treatment at 6 months, while only $8 \%$ patients were progression free after 8 months of treatment. At 16 months follow-up, our series showed a median OS of 9.2 months, with an interesting survival rate of 55.3, 32.9 and $22.4 \%$ at 8,12 and 16 months, respectively (Figure 2).

\section{Discussion}

The approval of trifluridine/tipiracil added a new important option in mCRC treatment. In fact, only two drugs (trifluridine/tipiracil and regorafenib) have evidence for a significant gain in OS against placebo in the difficult setting of patients with mCRC who have previously received all the available treatments, but with substantially higher toxicities profile [4]. No clinical comparison of these two drugs in monotherapy has been reported in terms 


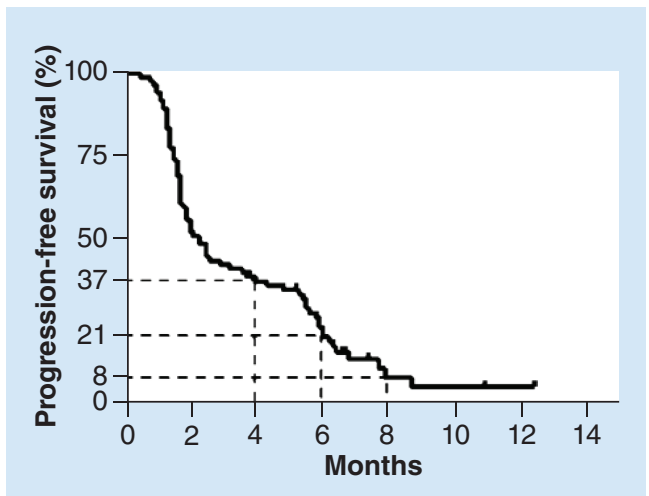

Figure 1. Progression-free survival.

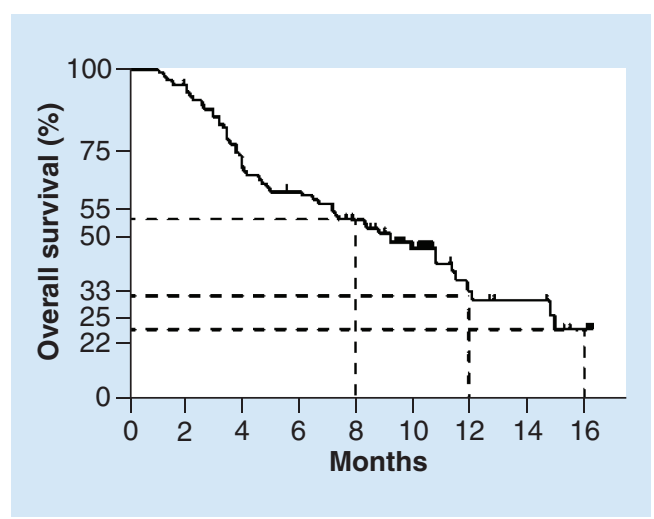

Figure 2. Overall survival.

of efficacy and safety, except for a Japanese trial of 74 patients treated with regorafenib or trifluridine/tipiracil as salvage-line therapy [26].

The pivotal trial, however, is fundamental to estimate the benefit provided by a new agent but often deviates from what is the clinical reality. The oncologist is left with a population that has different characteristics and needs than those of the studies. Topics such as AE management, efficacy out of trial selection criteria, decision on when is a better drug compared with other drug available in the same setting, and above all how to define the ideal patient for the treatment remains often unresolved by trials. In order to answer to these questions, we registered and analyzed data that report the use of trifluridine/tipiracil in real clinical practice in a cohort of 85 patients in our center.

Baseline characteristics of our patients' population were similar to those of patients enrolled in the pivotal trial. Differently from the pivotal trial, we have also enrolled in this series patients who had not received all conventional treatments, but that were deemed unfit or inappropriate for them with an overall 'less' pretreated population compared with the pivotal trial.

Our real life experience confirmed a mPFS of 2.3 months in an mCRC patient treated with trifluridine/tipiracil. Similar outcomes were reported in RECOURSE and TERRA study (mPFS of 2 months in both cases) [19]. Median OS was longer in our study (9.2 months) if compared with both Phase III results (7.1 and 7.8 months, respectively) regarding trifluridine/tipiracil treatment arm. Significantly, among patients who had disease stabilization with trifluridine/tipiracil, a small percentage of them were treated for several months, suggesting an interesting subgroup that could benefit in an impressive way from trifluridine/tipiracil.

Even the safety profile of trifluridine/tipiracil was in line with that of the pivotal study; AEs were mainly hematological: the most common $\mathrm{AE}$ was neutropenia, which had a relevant clinical impact causing treatment delays and, if it was recurrent or prolonged more than a week, dose reductions.

What seems quite impressive is the low incidence of febrile neutropenia, and the limited need to use G-CSF as secondary prophylaxis. Overall, the treatment was manageable, requiring permanent discontinuation of the treatment in one single case.

In our analysis, 21 patients were also treated with regorafenib before or after trifluridine/tipiracil. 
On the basis of current literature, trifluridine/tipiracil and regorafenib showed equivalent efficacy, but have different toxic profiles: neutropenia with trifluridine/tipiracil, whereas hyperbilirubinemia, skin disorders and hypertension were more common with regorafenib.

Until today, no clinical or biological factors could help us to choose the best treatment sequence among trifluridine/tipiracil and regorafenib but toxiciy profile could help physician's therapeutic choice, based on patient's clinical features, comorbidities and contraindications.

\section{Conclusion}

Results of our real-life experience confirmed the safety and efficacy profiles of trifluridine/tipiracil.

The toxicity profile had limited impact on patients' quality of life and could be clinically manageable, thus, trifluridine/tipiracil emerges as new valid option in chemorefractory mCRC treatment scenario, with a good risk/benefit rate.

\section{Future perspective}

At this time, trifluridine/tipiracil is a therapeutic chance for heavily pretreated and chemorefractory $\mathrm{mCRC}$ patients because of its safety profile and efficacy. Both published data and our data show this drug is well tolerated, with different and less severe toxicities than regorafenib, and with similar outcomes. Future challenges include to find biomarkers and other criteria in order to select patients and to choose the best sequence of trifluridine/tipiracil and regorafenib in each single case.

\section{Summary points}

- Chemorefractory metastatic colorectal cancer (mCRC) patients have restricted therapeutic chances and a poor prognosis.

- Clinical trials, regorafenib, trifluridine/tipiracil or best supportive care are the only options for these patients.

- Phase III trials, both with regorafenib and trifluridine/tipiracil monotherapy, have recently demonstrated an advantage in terms of efficacy if compared with placebo.

- On the basis of current literature, trifluridine/tipiracil and regorafenib showed equivalent efficacy, but have different toxic profiles.

- Clinical trial results provide the backbone of evidence-based medicine but they include an ideal selected population because of their restrictive eligibility criteria.

- It is fundamental to confirm randomized trials data in the real-life experience.

- A consecutive cohort of 85 heavily pretreated metastatic colorectal cancer received trifluridine/tipiracil in compassionate use in our institution.

- Our data showed similar outcomes if compared with data reported in Phase III trials.

- Even the safety profile of trifluridine/tipiracil was in line with drug toxicities reported in the pivotal study.

Author contributions

B Borelli, G Zucchelli, D Rossini, C Cremolini, C Antoniotti, R Moretto, F Marmorino, C Colombo, F Vannini, L Delliponti, E Ongaro, G Masi, IM Brunetti, E Pfanner, L Salvatore and A Falcone concepted and designed the article and performed collection and/or assembly of data. B Borelli, G Zucchelli, D Rossini, C Cremolini, C Antoniotti, R Moretto, F Marmorino, C Colombo, F Vannini, L Delliponti, M Spione, E Ongaro, G Masi, IM Brunetti, E Pfanner, L Salvatore and A Falcone did manuscript writing and performed the final approval of manuscript.

Financial \& competing interests disclosure

C Cremolini reports serving on advisory board for Roche and Merck Serono, and receiving grant support from Merck Serono; A Falcone reports serving on advisory board for Amgen, Merck Serono and Roche and receiving grant support from Amgen, Merck Serono and Roche. M Spione is a Servier employee. The authors have no other relevant affiliations or financial involvement with any organization or entity with a financial interest in or financial conflict with the subject matter or materials discussed in the manuscript apart from those disclosed.

Writing assistance funded by Servier was utilized in the production of this manuscript. 


\section{Ethical conduct of research}

The authors confirm that all the research meets the ethical guidelines, including adherence to the legal requirements of the study country - the study has been approved by the AOU Pisana Ethical Committee and was conducted according to the principles of the Declaration of Helsinki. They also state that for investigations involving human subjects, informed consent has been obtained from the participants involved.

\section{Open access}

This work is licensed under the CreativeCommons Attribution 4.0 License. To view a copy of this license, visit http://creativecomm ons.org/licenses/by/4.0/

\section{References}

Papers of special note have been highlighted as: $\bullet$ of interest; $\bullet \bullet$ of considerable interest

1. Van Cutsem E, Cervantes A, Nordlinger B, Arnold D; ESMO Guidelines Working Group. Metastatic colorectal cancer: ESMO Clinical Practice Guidelines for diagnosis, treatment and follow-up. Ann. Oncol. 25(Suppl. 3), iii1-iii9 (2014).

2. Beretta G, Salvatore L, Aprile G et al. Guidelines of the Italian Medical Oncology Association (AIOM) AIOM. www.aiom.it/C_Common/Download.asp?file=/\$Site\$/files/doc/LG/2017_LGAIOM_Colon.pdf

3. Benson AB 3rd, Venook AP, Cederquist L et al. Colon cancer, version 1.2017, NCCN Clinical Practice Guidelines in Oncology. J. Natl Compr. Cancer Netw. 15(3), 370-398 (2017).

4. Grothey A, Van Cutsem E, Sobrero A et al. Regorafenib monotherapy for previously treated metastatic colorectal cancer (CORRECT): an international, multicentre, randomised, placebo-controlled, Phase III trial. Lancet 381(9863), 303-312 (2013).

•• First Phase III evidence of a single-drug efficacy versus placebo in chemorefractory patients in the last few years.

5. Fukushima M, Suzuki N, Emura T et al. Structure and activity of specific inhibitors of thymidine phosphorylase to potentiate the function of antitumor 2'-deoxyribonucleosides. Biochem. Pharmacol. 59(10), 1227-1236 (2000).

6. Matsushita S, Nitanda T, Furukawa T et al. The effect of a thymidine phosphorylase inhibitor on angiogenesis and apoptosis in tumors. Cancer Res. 59(8), 1911-1916 (1999).

7. Temmink OH, Emura T, de Bruin M, Fukushima M, Peters GJ. Therapeutic potential of the dual-targeted TAS-102 formulation in the treatment of gastrointestinal malignancies. Cancer Sci. 98(6), 779-789 (2007).

8. Peters GJ, Backus HH, Freemantle S et al. Induction of thymidylate synthase as a 5-fluorouracil resistance mechanism. Biochim. Biophys. Acta 1587(2-3), 194-205 (2002).

9. Eckstein JW, Foster PG, Finer-Moore J, Wataya Y, Santi DV. Mechanism-based inhibition of thymidylate synthase by 5-(trifluoromethyl)-2'-deoxyuridine 5'-monophosphate. Biochemistry 33(50), 15086-15094 (1994).

10. Santi DV, Sakai TT. Thymidylate synthetase. Model studies of inhibition by 5-trifluoromethyl-2' -deoxyuridylic acid. Biochemistry 10(19), 3598-3607 (1971).

11. Emura T, Nakagawa F, Fujioka A et al. An optimal dosing schedule for a novel combination antimetabolite, TAS-102, based on its intracellular metabolism and its incorporation into DNA. Int. J. Mol. Med. 13(2), 249-255 (2004).

12. Emura T, Murakami Y, Nakagawa F, Fukushima M, Kitazato K. A novel antimetabolite, TAS-102 retains its effect on FU-related resistant cancer cells. Int. J. Mol. Med. 13(4), 545-549 (2004).

13. Emura T, Suzuki N, Yamaguchi M, Ohshimo H, Fukushima M. A novel combination antimetabolite, TAS-102, exhibits antitumor activity in FU-resistant human cancer cells through a mechanism involving FTD incorporation in DNA. Int. J. Oncol. 25(3), 571-578 (2004).

14. Yoshino T, Mizunuma N, Yamazaki K et al. TAS-102 monotherapy for pretreated metastatic colorectal cancer: a double-blind, randomised, placebo-controlled Phase II trial. Lancet Oncol. 13(10), 993-1001 (2012).

-• Fundamental basis for Phase III trials with trifluridin/tipiracil.

15. Overman MJ, Kopetz S, Varadhachary G et al. Phase I clinical study of three times a day oral administration of TAS-102 in patients with solid tumors. Cancer Invest. 26(8), 794-799 (2008).

16. Hong DS, Abbruzzese JL, Bogaard K et al. Phase I study to determine the safety and pharmacokinetics of oral administration of TAS-102 in patients with solid tumors. Cancer 107(6), 1383-1390 (2006).

17. Overman MJ, Varadhachary G, Kopetz S et al. Phase I study of TAS-102 administered once daily on a 5 day-per-week schedule in patients with solid tumors. Invest. New Drugs 26(5), 445-454 (2008).

18. Mayer RJ, Van Cutsem E, Falcone A et al. Randomized trial of TAS-102 for refractory metastatic colorectal cancer. N. Engl. J. Med. 372(20), 1909-1919 (2015).

- First randomized evidence of trifluridine/tipiracil effectiveness. 
19. Jianming Xu, Tae Won Kim, Lin Shen et al. Results of a randomized, double-blind, placebo-controlled, Phase III trial of trifluridine/tipiracil (TAS-102) monotherapy in asian patients with previously treated metastatic colorectal cancer: the TERRA Study. J. Clin. Oncol. 36(4), 350-358 (2017).

- Phase III data that confirm trifluridine/tipiracil feasibility and effectiveness even in Asian patients.

20. Rahbari M, Rahbari NN. Compassionate use of medicinal products in Europe: current status and perspectives. Bull. World Health Organ. 89(3), 163 (2011).

21. Patil S. Early access programs: Benefits, challenges, and key considerations for successful implementation. Perspect. Clin. Res. 7(1), 4-8 (2016).

22. Whitfield $\mathrm{K}$, Huemer $\mathrm{KH}$, Winter D et al. Compassionate use of interventions: results of a European Clinical Research Infrastructures Network (ECRIN) survey of ten European countries. Trials 11, 104 (2010).

23. Mishra D, Vora J. Non interventional drug studies in oncology: why we need them? Perspect. Clin. Res. 1(4), 128-133 (2010).

24. National Cancer Institute. Common Terminology Criteria for Adverse Events v4.0. May 29, 2009. https://ctep.cancer.gov/protocoldevelopment/electronic_applications/ctc.htm

25. Eisenhauer EA, Therasse P, Bogaerts J et al. New response evaluation criteria in solid tumours: revised RECIST guideline (version 1.1). Eur. J. Cancer 45(2), 228-247 (2009).

26. Kotaka M, Satake Y, Okita Y, Hatachi Y, Kotake T. Regorafenib vs TAS-102 as salvage-line treatment in patients with metastatic colorectal cancer refractory to standard chemotherapies: a multicenter retrospective comparison study. Ann. Oncol. 27(Suppl. 2), ii47 (2016) 